\title{
Isolation and Identification of Multi-drug resistant Pseudomonas aeruginosa from Burn Wound Infection in Chittagong City, Bangladesh
}

\author{
Pankqj Deb Nath ${ }^{1}$, Rocky Chowdhury ${ }^{1 *}$, Kartik Dhar ${ }^{1}$, Tumpa Dhar ${ }^{2}$, \\ Shoma Dutta ${ }^{1}$ \\ ${ }^{I}$ (Department of Microbiology, University of Chittagong, Chittagong 4331, Bangladesh) \\ ${ }^{2}$ (J. K. Memorial Hospital, Gahira, Raozan, Chittagong-4343, Bangladesh)
}

\begin{abstract}
Pseudomonas aeruginosa is an important infectious agent which causes nosocomial infections in immune-compromised patients, especially among burnt patients. In recent times, it has emerged as a far-flung Multi-Drug Resistant (MDR) pathogen which needs regular and periodic antibiotic susceptibility surveillance. The aim of this study was to isolate and identify MDR-P. aeruginosa from the burn patients held in the burn unit of Chittagong Medical College Hospital (CMCH) for $\sim 2$ months under treatment andto determine their antimicrobial resistance pattern for some commonly prescribed antibiotics. P. aeruginosa isolates were recovered from ten random wound swab samples of burnt patients through selective growth on MacConkey and Cetrimide agar media. Selected isolates were subjected to cultural, morphological and biochemical characterization. Antibiotic susceptibility patterns of the P. aeruginosa isolates were investigated by standard disk diffusion method of Kirby-Bauer. We assessed their antibiotic sensitivity patterns employing Amoxicillin, Ampicillin, Ceftazidime, Cephalexin, Chloramphenicol, Ciprofloxacin, Gentamicin, and Tetracycline. Two isolates of P. aeruginosa exhibited similar biochemical profiles but differ in cultural characteristics and antibiotypes; hence recognized as separate strains: P. aeruginosa CU1 and P. aeruginosa CU2. P. aeruginosa CU1 was found to be an MDR strain and showed resistance to all the test antibiotics. Not only the isolate exhibited resistance to ampicillin, amoxicillin, cephalexin, ciprofloxacin and tetracycline, but also showed remarkable resistance against two recommended antipseudomonal drugs: gentamicin and ceftazidime. Despite not being an MDR, P. aeruginosa CU2 exhibited resistance against amoxicillin, ampicillin and gentamicin. Though this report covered a small number of samplings and the results represent only a tiny fraction of total burnt patients of the Chittagong city; our findings are auguring enough considering public health concern which might spur care from public health management system of the nation.
\end{abstract}

Keywords: Burn wound, Multi-Drug Resistant, Pseudomonas aeruginosa

\section{Introduction}

$P$. aeruginosa is among the most common causes of severe infection in burnt patients [1,2]. Despite the variable degrees of burns, infection remains the most common cause of mortality amongst burnt patients [3]. Patients with open and large burn necrotic wounds are more vulnerable to $P$. aeruginosa infection $[4,5]$. $P$. aeruginosa is responsible for about $10 \%-20 \%$ of nosocomial infections as septicemia in the wound and burn infections, cystic fibrosis in intensive-care units (ICUs), etc.[6]. In nosocomial environments, strains of $P$. aeruginosa are the cause of several diseases, predominantly bacteremia, pneumonia, meningitis, urinary tract infections, and skin as well as soft-tissue infections [7]. P. aeruginosa has a preference for warm and moist environments of the wound, which is a remarkable dispute for burnt patients [8]. Moreover, antibiotic resistance made treatment of these infections frequently complicated, and this trouble is raising over time [9]. Regarding clinical importance, $P$. aeruginosa is mostly related to its prominent resistance to antimicrobial drugs, and also to its intrinsic resistance to antibiotics, becomes rapidly resistant to the drugs throughout the intervention [10].

An MDR- $P$. aeruginosa phenotype is determined with its resistant to more than two antipseudomonal antimicrobial classes; penicillins/cephalosporins, fluoroquinolones, carbapenems, and aminoglycosides [11]. Pseudomonas $s p$. is an omnipresent micro-organism that can quickly acquire resistance to various broadspectrum antibiotics. MDR-Pseudomonas $s p$. is a growing cause of morbidity and mortality in burnt patients. It causes $4 \%-60 \%$ nosocomial infections in different regions of the Earth [6]. The emergence of MDR strains among hospitalized burnt patients, especially in economically developing and underdeveloped nations, has become a remarkable trouble $[12,13]$.

$P$. aeruginosa isolates with significant resistance to antimicrobials have frequently been encountered in some tertiary heath care set up in Bangladesh [14]. There is a mounting manifest that proportion of resistance among $P$. aeruginosa isolates are rising gradually within Bangladeshi patients [15]. Although multi-drug resistance of the bacteria and the mechanisms contributing to this resistance have become a primary focus for 
researchers and health care practitioners worldwide, yet only a limited number of studies have been accomplished to discover MDR-P. aeruginosa in Bangladesh from burn wound infected patients.

The aim of this report was to isolate and identify MDR-P. aeruginosa from the burnt patients with prolonged hospitalization history in the Burn Unit of $\mathrm{CMCH}$. Two P. aeruginosa isolates were recovered from burn wound swab, and their susceptibility pattern against some antimicrobials had also been determined.

\subsection{Sampling}

\section{Methods And Material}

Surface swab samples were collected from Burn Unit of CMCH during June 2016. All the ten samples were collected from three third-degree deep burnt patients with prolonged ( $\sim 2$ months) hospitalization history. Swab samples from burn wounds were collected after removal of gauge bandage roll and before regular wound dressing. The tips of the swab cotton sticks were then transferred to sterile buffer peptone water tubes and transported to the laboratory.

\subsection{Isolation and identification of $P$. aeruginosa}

From each tube of the enriched buffer peptone water, one loopful culture was streaked across MacConkey agar surface. Plates were incubated for 24 hours at $37^{\circ} \mathrm{C}$. After incubation pale yellow coloured non-lactose fermenter colonies were isolated and purified. The suspected colonies were grown in separate flasks containing sterile Lauria-Bertani (LB) broth at $35^{\circ} \mathrm{C}$ for 24 hours. After incubation, one loopful broth from each LB culture was aseptically streaked across sterile pre-dried Cetrimide agar plates as a selective and differential medium for the isolation of $P$. aeruginosa. Then the inoculated plates were incubated at $35^{\circ} \mathrm{C}$ for 48 hours. Colonies on Cetrimide agar plates were then observed under short wavelength $(254 \mathrm{~nm})$ ultraviolet light for the presence of fluorescein. Biochemical and physiological behaviours were also investigated to confirm their identity.

\subsection{Antibiotic susceptibility test}

Antibiotic susceptibility tests for selected isolates were performed by disk diffusion technique of Kirby-Bauer [16]. The sensitivity of the isolates to antibiotics was assessed against commercially available standardized antibiotic discs of Amoxicillin $30 \mu \mathrm{g}$, Ampicillin $10 \mu \mathrm{g}$, Ceftazidime $30 \mu \mathrm{g}$, Cephalexin $30 \mu \mathrm{g}$, Chloramphenicol $30 \mu \mathrm{g}$, Ciprofloxacin $5 \mu \mathrm{g}$, Gentamicin $10 \mu \mathrm{g}$ and Tetracycline $30 \mu \mathrm{g}$. From subculture, the bacterial suspension was prepared in nutrient broth, and the turbidity of the culture was adjusted with the McFarland standard 0.5. A tipped cotton swab was dipped in the culture preparation and streaked over the surface of the Muller-Hinton agar medium to obtain uniform inoculum. The test-antibiotic discs were then placed on the surface of the seeded plates at appropriate arrangement by using sterile forceps, kept at $4^{\circ} \mathrm{C}$ for 30 minutes to diffuse antibiotic in the media and then the plates were incubated at $37^{\circ} \mathrm{C}$ for 24 hours. After incubation, the diameter of the clear zone of inhibition was measured, recorded and defined according to the standard tables given by the Clinical and Laboratory Standards Institute (CLSI) [17].

\section{Result And Discussion}

Our study focused on identification of MDR- $P$. aeruginosa from deep burn patients with a long hospital stay records. Identification started with the isolation of bacterial isolates from the samples and streaking them across MacConkey agar surfaces. Yellow coloured non-lactose fermenter colonies were suspected as $P$. aeruginosa and pyocyanin (a blue, water-soluble, non-fluorescent phenazine pigment) producing colonies with the characteristic grape-like odor of aminoacetophenone in Cetrimide agar plate were presumptively selected (Figure 1 and Figure 2).

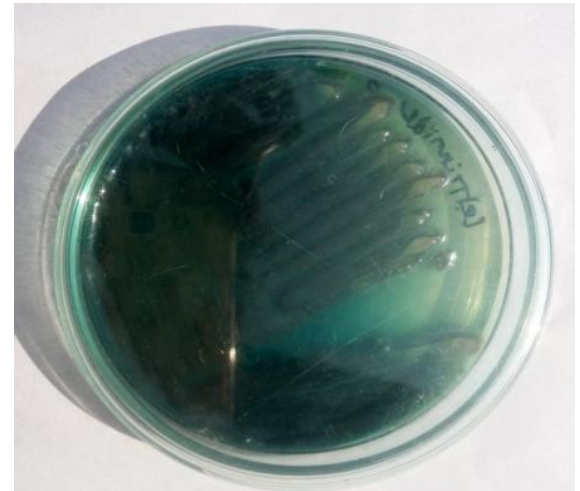

Fig. 1: Pyocyanin production of $P$. aeruginosa $\mathrm{CU} 1$ on Cetrimide agar plate 


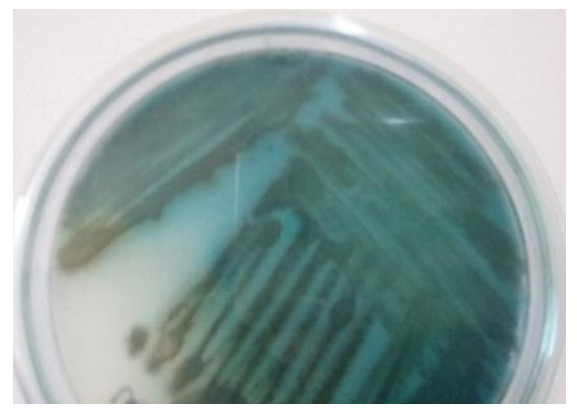

Fig. 2: Pyocyanin production of $P$. aeruginosa CU2 on Cetrimide agar plate

Only two isolates were selected for further study. Confirmatory identification was based on the cultural, morphological and biochemical behaviors of the presumptive isolates (Table 1).

Table 1: Physiological and Biochemical behavior of $P$. aeruginosa isolates

\begin{tabular}{|c|c|c|c|}
\hline \multirow[t]{2}{*}{ Category } & \multirow{2}{*}{ Test } & \multicolumn{2}{|c|}{ Behavior of the isolates } \\
\hline & & P. aeruginosa $\mathrm{CU} 1$ & P. aeruginosa $\mathrm{CU} 2$ \\
\hline \multirow{19}{*}{$\begin{array}{c}\text { Biochemical } \\
\text { behavior }\end{array}$} & Indole & - & - \\
\hline & MR & - & - \\
\hline & VP & - & - \\
\hline & Citrate & + & + \\
\hline & $\mathrm{H}_{2} \mathrm{~S}$ production & - & - \\
\hline & Nitrate reduction & + & + \\
\hline & Oxidase & + & + \\
\hline & Urease & + & + \\
\hline & Catalase & + & + \\
\hline & Gelatinase & + & + \\
\hline & Lipase & + & + \\
\hline & Acid from glucose & + & + \\
\hline & Gas from glucose & - & - \\
\hline & Lactose & - & - \\
\hline & Maltose & - & - \\
\hline & Mannitol & + & + \\
\hline & Sorbitol & - & - \\
\hline & Sucrose & - & - \\
\hline & Inulin & - & - \\
\hline \multirow{2}{*}{$\begin{array}{c}\text { Physiological } \\
\text { behavior }\end{array}$} & Motility & + & + \\
\hline & Growth at $4^{\circ} \mathrm{C}$ & - & - \\
\hline \multirow[t]{2}{*}{$\begin{array}{c}\text { Growth on selective } \\
\text { media }\end{array}$} & $\begin{array}{l}\text { Yellow colony on } \\
\text { MacConkey Agar }\end{array}$ & + & + \\
\hline & Pyocyanin production on Cetrimide Agar & + & + \\
\hline
\end{tabular}

"+” indicates positive result; "-" indicates negative result

Susceptibility of the target bacteria against some prescribed antibiotics was inquired. Between two $P$. aeruginosa isolates, $P$. aeruginosa CU1 was found to exhibit resistance against all representative antibiotics: $\beta$ lactams (ampicillin and amoxicillin), cephalosporins (cephalexin, ceftazidime), fluoroquinolone (ciprofloxacin), aminoglycosides (gentamicin) and tetracycline (Table 2).

Table 2: Antibiotic sensitivity patterns of the P. aeruginosa isolates against test antibiotics

\begin{tabular}{|c|c|c|c|c|c|c|c|c|}
\hline \multirow{3}{*}{ Antibiotic } & \multirow{3}{*}{$\begin{array}{l}\text { Antibiotic } \\
\text { Code }\end{array}$} & \multicolumn{7}{|c|}{ Susceptibility } \\
\hline & & \multicolumn{2}{|c|}{ P. aeruginosa CU1 } & \multicolumn{2}{|c|}{ P. aeruginosa CU2 } & \multicolumn{3}{|c|}{ Reference Value (mm) } \\
\hline & & Pattern & $\begin{array}{c}\text { Zone of } \\
\text { Inhibition } \\
(\mathrm{mm})\end{array}$ & Pattern & $\begin{array}{c}\text { Zone of } \\
\text { Inhibition } \\
(\mathrm{mm})\end{array}$ & $R^{I}$ & $I^{2}$ & $S^{3}$ \\
\hline Ciprofloxacin $(5 \mu \mathrm{g})$ & CIP-5 & $\mathrm{R}$ & 15 & $\mathrm{~S}$ & 25 & $\leq 15$ & $16-20$ & $\geq 21$ \\
\hline 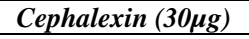 & LEX-30 & $\mathrm{R}$ & 0 & $\mathrm{~S}$ & 18 & $\leq 14$ & $15-17$ & $\geq 18$ \\
\hline Ampicillin $(10 \mu g)$ & AM-10 & $\mathrm{R}$ & 0 & $\mathrm{R}$ & 0 & $\leq 13$ & $14-16$ & $\geq 17$ \\
\hline Gentamicin $(10 \mu \mathrm{g})$ & GM-10 & $\mathrm{R}$ & 0 & $\mathrm{R}$ & 12 & $\leq 12$ & $13-14$ & $\geq 15$ \\
\hline Gentamicin $(10 \mu g)$ & $\mathrm{C}$ & $\mathrm{R}$ & 0 & $\mathrm{~S}$ & 26 & $\leq 12$ & $13-14$ & $\geq 15$ \\
\hline Tetracycline $(30 \mu \mathrm{g})$ & TE & $\mathrm{R}$ & 0 & $\mathrm{~S}$ & 23 & $\leq 11$ & $12-14$ & $\geq 15$ \\
\hline Amoxicillin (30 $\mu \mathrm{g})$ & $\mathrm{AmC}$ & $\mathrm{R}$ & 0 & $\mathrm{R}$ & 0 & $\leq 13$ & $14-17$ & $\geq 18$ \\
\hline Ceftazidime $(30 \mu \mathrm{g})$ & CAZ & $\mathrm{R}$ & 0 & $\mathrm{~S}$ & 21 & $\leq 14$ & $15-17$ & $\geq 18$ \\
\hline
\end{tabular}


Thus it is evident that $P$. aeruginosa CU1 was an MDR strain that had shown $\mathrm{CIP}^{\mathrm{R}} \mathrm{LEX}^{\mathrm{R}} A M^{\mathrm{R}} \mathrm{GM}^{\mathrm{R}} \mathrm{C}^{\mathrm{R}} \mathrm{TE}^{\mathrm{R}} A m C^{\mathrm{R}} C A Z^{\mathrm{R}}$ antibiotype. The result is surprising as well as threatening.

The resistance of $P$. aeruginosa against $\beta$-lactams and cephalosporins are well documented. Poole [18] attributed the resistance to $\beta$-lactams as a result of the yield of $\beta$-lactamase, active efflux of the antibiotics, or vitiated permeability. According to him resistance to $\beta$-lactams is because of the efflux mechanism belong to Resistance Nodulation Division (RND) family and may consist of a variety of efflux system. Other studies demonstrated high resistance rates of ceftazidime and other $3^{\text {rd }}$ generation cephalosporins like Benin [19]. The resistance of $P$. aeruginosa to the fluoroquinolone is also well documented [12]. Though resistance to ceftazidime is documented by several authors, it is still considered to be a good antipseudomonal drug. Gentamicin, another prescribed antipseudomonal drug, was found to fail to inhibit the growth of $P$. aeruginosa CU1. Resistance to aminoglycosides by $P$. aeruginosa is also not new [20]. However, resistance to ceftazidime and gentamicin in this study is an alarming finding.

$P$. aeruginosa CU2 was found to exhibit resistance against $\beta$-lactams (amoxicillin, ampicillin), and gentamycin (Table 2). The isolate had shown $\mathrm{CIP}^{\mathrm{S}} \mathrm{LEX}^{\mathrm{S}} \mathrm{AM}^{\mathrm{R}} \mathrm{GM}^{\mathrm{R}} \mathrm{C}^{\mathrm{S}} \mathrm{TE}^{\mathrm{S}} A m \mathrm{C}^{\mathrm{R}} \mathrm{CAZ} \mathrm{Z}^{\mathrm{S}}$ antibiotype. Though the isolate is not MDR, resistance to gentamicin is potentially threatening because, as stated above it is considered as one of the few drugs recommended to curb P. aeruginosa infection.

\section{Conclusion}

Two isolates of $P$. aeruginosa were recovered from burn wound infection. One isolate showed multidrug resistance and another exhibited remarkable resistance against an antipseudomonal drug like gentamicin. Resistance pattern against test antibiotics showed by the isolates omen onset of serious diseases once the organisms find their way toward infection. The study urges proper revision of wound care procedures as well as maintenance of hygienic conditions in burn care unit to prevent a nosocomial outbreak of MDR- $P$. aeruginosa. Also, a high percentage of resistance to antibiotics indicates the improper use of antibiotics in the hospital. Isolates' DNA Sequencing, antibiogram to other recommended antipseudomonal drugs, and insight into the drug resistance mechanism is planned to be carried out toward characterization of these two formidable $P$. aeruginosa isolates.

\section{Acknowledgements}

The authors like to convey their thanks to The University of Chittagong, Chittagong 4331, Bangladesh for financial support.

\section{References}

[1] Eltahawy, A.T., Gram-negative bacilli isolated from patients in intensive care unit: prevalence and antibiotic susceptibility. J Chemother, 1997. 9(6): p. 403-10.

[2] Mousa, H.A., Aerobic, anaerobic and fungal burn wound infections. J Hosp Infect, 1997. 37(4): p. 317-23.

[3] Saffle, J.R., B. Davis, and P. Williams, Recent outcomes in the treatment of burn injury in the United States: a report from the American Burn Association Patient Registry. J Burn Care Rehabil, 1995. 16(3 Pt 1): p. 219-32; discussion 288-9.

[4] Lister, P.D., D.J. Wolter, and N.D. Hanson, Antibacterial-resistant Pseudomonas aeruginosa: clinical impact and complex regulation of chromosomally encoded resistance mechanisms. Clin Microbiol Rev, 2009. 22(4): p. 582-610.

[5] Rastegar Lari, A.R., R. Alaghehbandan, and L. Akhlaghi, Burn Wound Infections and Antimicrobial Resistance in Tehran, Iran: an Increasing Problem. Annals of Burns and Fire Disasters, 2005. 18(2): p. 68-73.

[6] Carmeli, Y., et al., Emergence of Antibiotic-Resistant Pseudomonas aeruginosa: Comparison of Risks Associated with Different Antipseudomonal Agents. Antimicrobial Agents and Chemotherapy, 1999. 43(6): p. 1379-1382.

[7] Wróblewska, M., Novel therapies of multidrug-resistant Pseudomonas aeruginosa and Acinetobacter spp . infections: the state of the art. Archivum Immunologiae et Therapiae Experimentalis, 2006. 54(2): p. 113-120.

[8] Hassuna, N.A., et al., High Prevalence of Multidrug Resistant Pseudomonas aeruginosa Recovered from Infected Burn Wounds in Children. Archives of Clinical Microbiology, 2015. 6(4).

[9] Nakae, M., et al., Serotypes and drug susceptibility of Pseudomonas aeruginosa isolated from clinical specimens. Jpn J Antibiot, 1997. 50(2): p. 187-94.

[10] Tsutsui, A., et al., Genotypes and infection sites in an outbreak of multidrug-resistant Pseudomonas aeruginosa. J Hosp Infect, 2011. 78(4): p. 317-22.

[11] Magiorakos, A.P., et al., Multidrug-resistant, extensively drug-resistant and pandrug-resistant bacteria: an international expert proposal for interim standard definitions for acquired resistance. Clin Microbiol Infect, 2012. 18(3): p. 268-81.

[12] Japoni, A., S. Farshad, and A. Alborzi, Pseudomonas aeruginosa: Burn Infection, Treatment and Antibacterial Resistance. Iran Red Crescent Med J, 2009. 11(3): p. 244-253.

[13] Moniri, R., et al., Emergence of multi-drug-resistant Pseudomonas aeruginosa isolates in neonatal septicemia. Journal of Infectious Diseases and Antimicrobial Agents, 2005. 22(2): p. 39-44.

[14] Begum, S., et al., Detection of extended spectrum $\beta$-lactamase in Pseudomonas spp. isolated from two tertiary care hospitals in Bangladesh. BMC Research Notes, 2013. 6(1): p. 7.

[15] Rashid, A., et al., Infections by Pseudomonas aeruginosa and antibiotic resistance pattern of the isolates from Dhaka Medical College Hospital. Bangladesh Journal of Medical Microbiology, 2016. 1(2): p. 48-51.

[16] Bauer, A.W., et al., Antibiotic susceptibility testing by a standardized single disk method. Am J Clin Pathol, 1966. 45(4): p. 493-6.

[17] Cockerill, F.R., Performance standards for antimicrobial susceptibility testing: twenty-first informational supplement. 2011: Clinical and Laboratory Standards Institute (CLSI). 
Isolation and Identification of Multi-drug resistant Pseudomonas aeruginosa from Burn Wound Infection in Chittagong City, Bangladesh

[18] Poole, K., Efflux-mediated antimicrobial resistance. J Antimicrob Chemother, 2005. 56(1): p. 20-51.

[19] Ahoyo, T.A., et al., Prevalence of nosocomial infections and anti-infective therapy in Benin: results of the first nationwide survey in 2012. Antimicrobial Resistance and Infection Control, 2014. 3: p. 17-17.

[20] Kamaria, P.A., B.J. Aring, and M. Sinha, Incidence of Multidrug Resistant Pseudomonas Aeruginosa Isolated From Burn Patients Tertiary Care Hospital, Jamnagar, Gujarat, India. IOSR Journal of Dental and Medical Sciences (IOSR-JDMS), 2016. 1(15): p. $31-34$. 